\title{
Impairment of quality of life and cognition in demyelinating neuropathies: targets to be considered from diagnosis to treatment?
}

\author{
Comprometimento da qualidade de vida e da cognição em neuropatias desmielinizantes: \\ alvos a serem considerados do diagnóstico ao tratamento?
}

Osvaldo J. M. Nascimento

\section{MD, PhD, Full Professor of Neurology, Departamento de Neurologia, Núcleo de Pesquisa em Neurologia/Neurociências do Hospital Universitário Antonio Pedro, Universidade Federal Fluminense, Rio de Janeiro RJ - Brazil.}

Correspondence:

Osvaldo JM Nascimento

Rua Siqueira Campos 53/1204,

Copacabana; 22031-071

Rio de Janeiro RJ - Brasil

E-mail: osvaldo_nascimento@

hotmail.com

Conflict of interest:

There is no conflict of interest to declare.

Received 13 January 2014 Accepted 21 January 2014

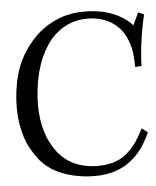

hronic inflammatory demyelinating diseases (CIDD) comprise a spectrum of disorders affecting the myelin of central (CNS) and peripheral nervous system (PNS). These disorders can usually be differentiated on the basis of clinical, imaging, laboratory, neurophysiological and pathological findings. Quality of life (QoL) measurements are being considered increasingly important with regard to evaluate disease progression, treatment and the management of care provided to neurological patients with these diseases. Chronic inflammatory demyelinating polyradiculoneuropathy (CIDP) is a sensorimotor neuropathy that evolves over at least 2 months with either a progressive or a relapsing remitting course. CIDP includes a broad spectrum of clinical phenotypes, and their recognition is important because of varied treatment responses ${ }^{1}$. Various diagnostic criteria have been proposed for CIDP. Recently the European Federation of Neurological Societies (EFNS)/PNS guidelines have taken also into consideration the response to immunomodulatory treatment ${ }^{2}$.

The use of parametric instruments, including the biopsychosocial assessment is proving to be very important throughout PNS demyelinating disease evolution ${ }^{3}$, as also recently happens with CNS demyelinating diseases such as multiple sclerosis (MS). QoL together with cognition have received special attention in the management of MS patients ${ }^{4}$. The inclusion of QoL among clinical assessment instruments allows a better therapeutic approach. In this issue of Arquivos de Neuro-Psiquiatria, Santos and colleagues ${ }^{5}$ offer a very important contribution reporting the psychological profile and QoL associated to CIDP, studying the Mini-Mental State Examination (MMSE) and the Short Form Health Survey (SF36) in 41 Brazilian patients. Impaired functional capacity and pain were the most important alterations in the health status. The authors concluded that these impairments resulted into professional and personal limitations. They emphasize a previous report suggesting the importance of QoL evaluation in the knowledge of the evolution and prognosis of $\mathrm{CIDP}^{3}$. We consider that it is very important to, specifically and separately, assess each CIDP variant, taking into account that each one has distinctive treatment responsiveness, suggesting varied underlying pathophysiologic mechanisms.

There was a high $(29.3 \%)$ prevalence of diabetes in the CIDP series of Santos et al. ${ }^{5}$. We presume to be prudent including these patients into a separate group due to the close association of diabetes with QoL reduction. Pain is the most common cause of functional impairment in the clinical practice, commonly seen in axonal neuropathies. Pain was a complaint in $48.8 \%$ of patients in Santos et al. ${ }^{5}$ series. A very interesting presentation of CIDP with only painful symptoms, most in the distal legs and responsive to immunoglobulin has been reported ${ }^{6}$. A QoL assessment in these patients should be very important in the followup. Pain and difficulty in walking are very frequent complaints resulting in a negative socioeconomic impact to the patient. Functional capacity has been evaluated in CIDP patients, but only in Santos et al. ${ }^{5}$ report we found information about work activity, and leisure reduction during the disease. 
CIDD if not properly diagnosed and treated result in axonal loss of greater or lesser severity favoring the development of associated symptoms. This process remains unclear. However, it seems that a persistent inflammatory/ immune state would be the determinant. Some recent reports try to clarify this question. As examples, in CIDP patients, regulatory $\mathrm{T}$ cells display a defect of suppressive function ${ }^{7}$, and in the uncommon combined central and peripheral demyelination cases anti-neurofascin antibody is frequently present ${ }^{8}$. Among the various associated signs and symptoms involved in the functional evaluation of patients with demyelinating diseases, two have called attention: fatigue and impaired cognition. One-third (34.1\%) of Santos et al. ${ }^{5}$ patients complained of weakness, fatigue or tiredness at the time of evaluation, showing that they are frequent. Thus, fatigue should be considered when assessing psychological profile and QoL in CIDP and variants.

During the last two decades neurologists and psychologists have become increasingly aware of the prevalence and functional impact of the association of MS and cognitive impairment (CI). Recent studies have highlighted that CI should be looked for, regardless of the disease duration or clinical course ${ }^{9}$. Santos et al. ${ }^{5}$ report memory deficit in $34.1 \%$ of their CIDP patients, bringing the question about the association of CI and CIDP. Studies with proper instruments focusing the presence of $\mathrm{CI}$ in demyelinating neuropathies, regarding the relationship with duration and clinical course, could be interesting. It is very important to exclude associated health problem as diabetes, arterial hypertension, hypothyroidism and others, referred in $65.9 \%$ of cases in Santos et al series ${ }^{5}$.

Considering the socioeconomic profile and QoL is important in the evaluation of effective new therapeutic perspectives. In this sense, limited evidence suggests that the subcutaneous route for immunoglobulin therapy may be a more patient-friendly option than the traditional intravenous alternative ${ }^{10}$.

The elegant report of Santos et al. ${ }^{5}$ brought some reflections about the biopsychosocial profile of CIDP patients. Thus, the QoL and cognition impairments need to be better known in demyelinating neuropathies, being possible targets to be considered in the diagnosis, treatment and prognosis of CIDP and its variants.

\section{References}

1. Eftimov F, van Schaik I. Chronic inflammatory demyelinating polyradiculoneuropathy: update on clinical features, phenotypes and treatment options. Curr Opin Neurol 2013;26:496-502.

2. European Federation of Neurological Societies/Peripheral Nerve Society Guideline on management of chronic inflammatory demyelinating polyradiculoneuropathy: report of a joint task force of the European Federation of Neurological Societies and the Peripheral Nerve Society-First Revision. J Peripher Nerv Syst 2010;15:1-9.

3. Merkies ISJ, Hughes RAC, Donofrio P, et al. Understanding the consequences of chronic inflammatory demyelinating polyradiculoneuropathy from impairments to activity and participation restrictions and reduced quality of life: the ICE study. J Peripher Nerv Syst 2010;15:208-215.

4. Goretti B, Portaccio E, Zipoli V, Razzolini L, Amato MP. Coping strategies, cognitive impairment, psychological variables and their relationship with quality of life in multiple sclerosis. Neurol Sci 2010;31(Suppl 2):S227-S230.

5. Santos PL, Ribeiro GAN, Silva DMD, Marques Jr W, Barreira AA. Chronic inflammatory demyelinating polyneuropathy: quality of life, sociodemographic profile and physical complaints. Arq Neuropsiquiatr 2014;72.

6. Nascimento OJM, Freitas MRG, Escada TM. Painful sensory polineuropathy responsive to immunoglobulin: a sensory CIDP variant? J Neurol 2006;253(Suppl):S12-S13.

7. Sanvito L, Makowska A, Gregson N, Nemni R, Hughes RA. Circulating subsets and $\mathrm{CD} 4(+) \mathrm{CD} 25(+)$ regulatory $T$ cell function in chronic inflammatory demyelinating polyradiculoneuropathy. Autoimmunity 2009;42:667-677.

8. Kawamura N, Yamasaki R, Yonekawa T, et al. Anti-neurofascin antibody in patients with combined central and peripheral demyelination. Neurology 2013;81:714-722.

9. Amato MP, Portaccio E, Goretti B, et al. Cognitive impairment in early stages of multiple sclerosis. Neurol Sci 2010;31(Suppl 2):S211-S214.

10. Rajabally YA. Subcutaneous immunoglobulin therapy for inflammatory neuropathy: current evidence base and future prospects. J Neurol Neurosurg Psychiatry 2013;0:1-7. Doi: 10.1136/jnnp-2013-305644. 